\title{
Association between Academic Learning Strategies and Annual Examination Results among Medical Students of King Edward Medical University
}

\author{
Saira Tariq, ${ }^{1}$ Muhammad Arif Khan, ${ }^{2}$ Saira Afzal, ${ }^{3}$ Sheikh Raza Shahzad, ${ }^{4}$ Mohammad Hamza \\ Haania Aimen Khan, ${ }^{6}$ Fatima Qamar, ${ }^{7}$ Maryam Javed, ${ }^{8}$ Muhammad Farhan Ashraf, ${ }^{9}$ Fareeha Mubarak ${ }^{10}$ \\ BushraIqbal Puri, ${ }^{11}$ Taha Masood, ${ }^{12}$ Fatima Kazim, ${ }^{13}$ Tanveer Shaukat, ${ }^{14}$ Shafia Shaikh ${ }^{15}$
}

\begin{abstract}
Learning style is the composite of cognitive, affective, and physiological characteristics that serve as relatively stable indicators of how a learner perceives, interacts and responds to the learning environment. Felder - Solomon Index of Learning Style (ILS) includes Sensitive/Intuitive, Visual/Verbal and Sequential/ Global, Active/reflective. The learning strategies are a major indicator of exam performance of students.

Objective: To find the association between academic
\end{abstract}

Tariq $S .^{1}$

Assistant Professor, Department of Community Medicine

King Edward Medical University, Lahore

Khan M.A. ${ }^{2}$

APMO, Department of Community Medicine

King Edward Medical University, Lahore

Afzal S. ${ }^{3}$

Chairperson and Head of Community Medicine

King Edward Medical University, Lahore

Shahzad S.R. ${ }^{4}$

MBBS Student, King Edward Medical University, Lahore

Hamza M. ${ }^{5}$

MBBS Student, King Edward Medical University, Lahore

Khan H.A. ${ }^{6}$

MBBS Student, King Edward Medical University, Lahore

Qamar F.

MBBS Student, King Edward Medical University, Lahore learning strategies and annual examination results among medical students of King Edward Medical University.

Date of Submission 1-2-2016

Date of Acceptance for Publication 3-5-2016

Conflict of Interest: None

Funding Source: None
Javed M. ${ }^{8}$

MBBS Student, King Edward Medical University, Lahore

Ashraf M.F. ${ }^{9}$

MBBS Student, King Edward Medical University, Lahore

Mubarak F. ${ }^{10}$

MBBS Student, King Edward Medical University, Lahore

Puri B.I. ${ }^{11}$

MBBS Student, King Edward Medical University, Lahore

Masood T. ${ }^{12}$

MBBS Student, King Edward Medical University, Lahore

Kazim F. ${ }^{13}$

MBBS Student, King Edward Medical University, Lahore

Shaukat $\mathrm{T}^{14}$

MBBS Student, King Edward Medical University, Lahore

Shaikh S. ${ }^{15}$

MBBS Student, King Edward Medical University, Lahore 
Study Design: Cross - sectional study design.

Study Setting and Duration: King Edward Medical University and 3 months.

Materials and Methods: The study was conducted among the $3^{\text {rd }}, 4^{\text {th }}$, and $5^{\text {th }}$ year medical students of KEMU Lahore. Informed consent was taken. A total of 300 students, 100 each from $3^{\text {rd }}, 4^{\text {th }}$ and $5^{\text {th }}$ year were taken. 132 males (44\%) and 168 females (56\%) were part of the study. Simple Random sampling (non-probability sampling) was used.

Results: The overall dominant learning strategies among students of King Edward Medical University were sensing/SNS (64.3\%), visual/VIS (80.7\%), sequential/SEQ (63.7\%) and Active/ACT (50.3\%). Chi squared test for individual learning combinations against achiever types showed no significant association between academic learning strategies and annual exam performance ( $\mathrm{p}$ value $>0.05$ ). Results also showed that females performed better academically than their male counterparts.

Keywords: Learning strategies, Index of learning styles, exam performances, medical students.

\section{Introduction}

"Learning style is the composite of cognitive, affective, and physiological characteristics that serve as relatively stable indicators of how a learner perceives, interacts and responds to the learning environment". Student oriented learning has taken precedence over teacher oriented one. This has resulted in adoption of new teaching methods that allow students to employ their own methodology. ${ }^{2}$ The learning style of a student is a major indicator of his performance in examinations. Students with different learning styles perform differently on different exam questions. ${ }^{3}$ Various studies have been conducted in the past in order to establish the spectrum of learning styles adopted by students. Till date there has been no study carried out to establish the relationship between exam performance and learning styles employed by students.

Different researchers have developed different methods to qualify and quantify different learning strategies. VARK developed by Fleming ${ }^{4}$ describes students' preference for Visual (V), Aural (A), Read/ Write (R) and Kinesthetic (K); it tells us which teaching style is the most conducive to a student's learning in a particular setting. Index Learning Style (ILS) is an instrument developed by Felder and Silverman ${ }^{5}$ which is used to assess preferences on four dimensions ${ }^{6}$ of a learning style model. These four dimensions are: Processing: Active/Reflective, Perception: Sensing/Intuitive, Input: Visual/Verbal, Understanding: Sequential/ Global. Vermunt ${ }^{7}$ developed a method spanning four dimensions of cognitive processing, metacognitive regulation, mental learning models and learning orientations. Based on these characteristics, he classified student learning styles as undirected, reproduction directed, meaning - directed and application - directed. We shall use ILS model in our study because VARK has little to say about personality, motivation, social preferences, physical environment or intraversion - extraversion and also because it inclines more towards teaching directed than self - directed learning. ILS identifies Vermont's four learning styles and is a more practical in use. An individual learning style is subject to variation with time, learning environment and course enrollment. The learning preponderance of an engineering major may not be the same as that of a medical student. A student's inclination towards a particular learning dimension provides an insight into his strengths, habits and tendencies which might affect his performance in academic set up. ILS is designed to assess preference on the four dimension of Felder Silverman learning style. Based on ILS model, a study conducted in 2003 on 493 first year engineering students of Aalborg University, Denmark revealed that the majority was active, visual and sensing type while sequential and global learners were relatively equal. ${ }^{8}$ Furthermore, among 129 Materials engineering majors at Iowa State university, $63 \%$ were active, $67 \%$ sensing type, $85 \%$ visual and $58 \%$ sequential learners. Undergraduates totaling 2506 from various engineering disciplines and from across different universities were tested for the preponderance of learning strategies. Active (64\%); Sensing (63\%); visual (82\%) and sequential $(60 \%)$ were the dominant preferences. A relative mismatch was observed between the engineering undergraduates' learning preferences and the teaching styles. Teaching methodology was more inclined towards reflective, intuitive and global learning.

In the past, the predominant learning style in medical students has been found. ${ }^{9}$ However, to date, no research of sorts has been conducted regarding correlation of exam scores and learning styles of medical students particularly in the context of Pakistan. The rationale of this study is to establish the preponderance of learning strategies according to the ILS questionnaire amongst the students of King Edward Medical University and its association with exam performance.

This research aims to investigate the preponde- 
rance of learning strategies among under - achievers, achievers, high achievers and overachievers. It will help form a comparison between the different strategies adopted and establish the superiority of the learning strategies of high - achievers, which shall lead to the establishment of a successful pattern of academic learning styles that could revolutionize the exam results and curb the social problems arising due to failure. Additionally, this could call for a more uniform question - writing in exams and for instructors to have a better match between their teaching styles and students' needs.

\section{Materials and Methods}

The study was conducted among the $3^{\text {rd }}, 4^{\text {th }}$, and $5^{\text {th }}$ year medical students of KEMU Lahore. Informed consent was taken. A total of 300 students, 100 each from $3^{\text {rd }}, 4^{\text {th }}$ and $5^{\text {th }}$ year were taken. 132 males $(44 \%)$ and 168 females $(56 \%)$ were part of the study. Cluster sampling (probability sampling) was used. Excluded from our study were:

- Students of Allied Health sciences/MD/MS programs.

- Students from other colleges.

- Students having any physical disability.

- Students having any mental illness.

- Students other than $3^{\text {rd }}, 4^{\text {th }}$ and final year MBBS.

- Non cooperative students.

\section{Study Design}

Cross sectional study.

\section{Study Setting and Duration}

King Edward Medical University and 3 month duration.

\section{Data Collection Procedure}

Data is pretested. A total of $3003^{\text {rd }}, 4^{\text {th }}$ and final year MBBS students were asked to fill out the bio - data pro forma and Felder - Solomon Index of Learning Style (ILS) questionnaire manually. Data was collected by all our team members. According to ILS questionnaire, each participant is given 44 questions, 11 from each dimension of learning style with a possible ' $a$ ' or ' $b$ ' answer. The scores of ' $b$ ' in each dimension are subtracted from scores of ' $a$ ' in the same dimen- sion to reach an integer between -11 to +11 which indicates both the learning strategy and its strength. The inclination towards a particular style maybe mild, moderate or strong.

\section{Operational Definitions}

\section{Exam Performance}

- Under - achievers: Scoring less than 65\% in their exams (mean of all professional exam scores).

- Achievers: Scoring $65 \%$ or above and less than $70 \%$ in their exams (mean of all professional scores).

- High - achievers: Scoring 70\% or above and less than $75 \%$ in their exams (mean of all professional scores).

- Overachievers: Scoring $75 \%$ or above (mean of all professional scores).

\section{Learning Strategies}

There were 4 pairs of learning strategies in total which are as follows:

\section{Sensing/Intuitive:}

- Sensing (SNS): Concrete thinker, practical, oriented towards facts and procedures.

- Intuitive (INT): Abstract thinker, innovative, oriented towards theories and underlying meanings.

\section{Visual/Verbal}

- Visual (VIS): Prefer visual representation of presented material such as pictures, diagrams and flow charts

- Verbal (VRB): Prefer written or spoken explanation.

\section{Active/Reflective}

- Active (ACT): Learn by trying things out, enjoy working in groups.

- Reflective (REF): Learn by thinking things through; prefer working alone or with a single familial partner.

\section{Sequential/Global}

- Sequential: Liner thinking process, learn in small increment steps. 
- Global: Holistic thinking process, learn in large leaps.

\section{Hypothesis}

There exists an association between academic learning strategies and annual examination results among medical students of King Edward Medical University.

\section{Null Hypothesis}

There is no association between academic learning strategies and annual examination results among medical students of King Edward Medical University.

\section{Data Analysis}

All collected data was entered and analyzed in computer program SPSS version 22. Frequency and percentage of dominant learning strategies among overachievers, high achievers, achievers and underachievers was calculated. Inter - gender and inter - class comparison was also a part of the study. The $p$ value $\leq 0.05$ was considered significant. At $\mathbf{d f}=\mathbf{3}$ and $\mathrm{p} \leq 0.05 ; \mathrm{X}^{2}{ }_{\text {crit }}=7.81$. If $\mathrm{X}_{\text {calc }}{ }$ is more than 7.81 , result becomes significant.

\section{Results}

\section{Examination Results and Academic Learning Strategies}

The overall dominant learning strategies among stu- dents of King Edward Medical University were sensing/SNS (64.3\%), visual/VIS (80.7\%), sequential/SEQ (63.7\%) and Active/ACT (50.3\%). Majority of the overachievers were Reflective (REF), Visual (VIS) and Sequential (SEQ). The number of Sensing (SNS) and Intuitive (INT) learners among overachievers was the same. High achievers had the greatest proportion of Active learners compared to the other three achiever types. High achievers showed preponderance for active, sensing, visual and sequential learning strategies. While majority of high achievers were of the Active type, the greatest proportion of overachievers was of the Reflective type. Achievers were mainly reflective, sensing, visual and sequential. As far as the underachievers are concerned, majority were of the Reflective, Sensing, Visual and Sequential types. The most common learning strategy from the four learning combinations in all achiever types was Visual. Chi squared test for individual learning combinations against achiever types showed no significant association between academic learning strategies and annual exam performance $(p$ value $>0.05)$.

\section{Gender Preponderance}

An analysis drawn between gender and learning types revealed that more males were Reflective, Sensing, Visual and Sequential while females only differed from their male counterparts in being more Active.

Furthermore, our study shows that significant statistical difference exists between exam performances of females and males. Females out - perform their male counterparts.

\section{Percentages ACT/REF}

\begin{tabular}{|c|c|c|c|c|c|}
\hline \multicolumn{2}{|c|}{} & Frequency & Percent & Valid Percent & Cumulative Percent \\
\hline \multirow{3}{*}{ Valid } & ACT & 151 & 50.3 & 50.3 & 50.3 \\
\cline { 2 - 6 } & REF & 149 & 49.7 & 49.7 & 100.0 \\
\cline { 2 - 6 } & Total & 300 & 100.0 & 100.0 & \\
\hline
\end{tabular}

\section{SNS/INT}

\begin{tabular}{|c|l|c|c|c|c|}
\hline \multicolumn{2}{|c|}{} & Frequency & Percent & Valid Percent & Cumulative Percent \\
\hline \multirow{3}{*}{ Valid } & SNS & 193 & 64.3 & 64.3 & 64.3 \\
\cline { 2 - 6 } & INT & 107 & 35.7 & 35.7 & 100.0 \\
\cline { 2 - 6 } & Total & 300 & 100.0 & 100.0 & \\
\hline
\end{tabular}


VIS/VRB

\begin{tabular}{|c|c|c|c|c|c|}
\hline \multicolumn{2}{|c|}{} & Frequency & Percent & Valid Percent & Cumulative Percent \\
\hline \multirow{3}{*}{ Valid } & VIS & 242 & 80.7 & 80.7 & 80.7 \\
\cline { 2 - 6 } & VRB & 58 & 19.3 & 19.3 & 100.0 \\
\cline { 2 - 6 } & Total & 300 & 100.0 & 100.0 & \\
\hline
\end{tabular}

\section{SEQ/GLO}

\begin{tabular}{|c|c|c|c|c|c|}
\hline \multicolumn{2}{|c|}{} & Frequency & Percent & Valid Percent & Cumulative Percent \\
\hline \multirow{4}{*}{ Valid } & SEQ & 191 & 63.7 & 63.7 & 63.7 \\
\cline { 2 - 6 } & GLO & 109 & 36.3 & 36.3 & 100.0 \\
\cline { 2 - 6 } & Total & 300 & 100.0 & 100.0 & \\
\hline
\end{tabular}

\section{Achiever type}

\begin{tabular}{|c|l|c|c|c|c|}
\hline \multicolumn{2}{|c|}{} & Frequency & Percent & Valid Percent & Cumulative Percent \\
\hline \multirow{4}{*}{ Valid } & Overachiever & 16 & 5.3 & 5.3 & 5.3 \\
\cline { 2 - 6 } & High achiever & 101 & 33.7 & 33.7 & 39.0 \\
\cline { 2 - 6 } & Achiever & 122 & 40.7 & 40.7 & 79.7 \\
\cline { 2 - 6 } & Underachiever & 61 & 20.3 & 20.3 & 100.0 \\
\cline { 2 - 6 } & Total & 300 & 100.0 & 100.0 & \\
\hline
\end{tabular}

\section{COMPARISON}

\begin{tabular}{|c|c|c|c|c|c|}
\hline & & & \multicolumn{2}{|c|}{$\mathrm{ACT} / \mathrm{REF}$} & \multirow{2}{*}{ Total } \\
\hline & & & $\mathrm{ACT}$ & REF & \\
\hline \multirow{8}{*}{ Achiever type } & \multirow{2}{*}{ Overachiever } & Count & 6 & 10 & 16 \\
\hline & & $\%$ within Achiever type & $37.5 \%$ & $62.5 \%$ & $100.0 \%$ \\
\hline & \multirow{2}{*}{ High achiever } & Count & 59 & 42 & 101 \\
\hline & & $\%$ within Achiever type & $58.4 \%$ & $41.6 \%$ & $100.0 \%$ \\
\hline & \multirow{2}{*}{ Achiever } & Count & 58 & 64 & 122 \\
\hline & & $\%$ within Achiever type & $47.5 \%$ & $52.5 \%$ & $100.0 \%$ \\
\hline & \multirow{2}{*}{ Underachiever } & Count & 28 & 33 & 61 \\
\hline & & $\%$ within Achiever type & $45.9 \%$ & $54.1 \%$ & $100.0 \%$ \\
\hline \multirow{2}{*}{\multicolumn{2}{|c|}{ Total }} & Count & 151 & 149 & 300 \\
\hline & & $\%$ within Achiever type & $50.3 \%$ & $49.7 \%$ & $100.0 \%$ \\
\hline
\end{tabular}


Chi-Square Tests

\begin{tabular}{|l|c|c|c|}
\hline & Value & Df & $\begin{array}{c}\text { Asymp. Sig. } \\
(2 \text {-sided })\end{array}$ \\
\hline Pearson Chi-Square & $2.964^{\mathrm{a}}$ & 3 & .397 \\
\hline Likelihood Ratio & 2.902 & 3 & .407 \\
\hline N of Valid Cases & 300 & & \\
\hline
\end{tabular}

a. 0 cells $(.0 \%)$ have expected count less than 5. The minimum expected count is 5.71 .

The $\mathbf{X}^{2}$ calc is less than 7.81 so result is statistically insignificant

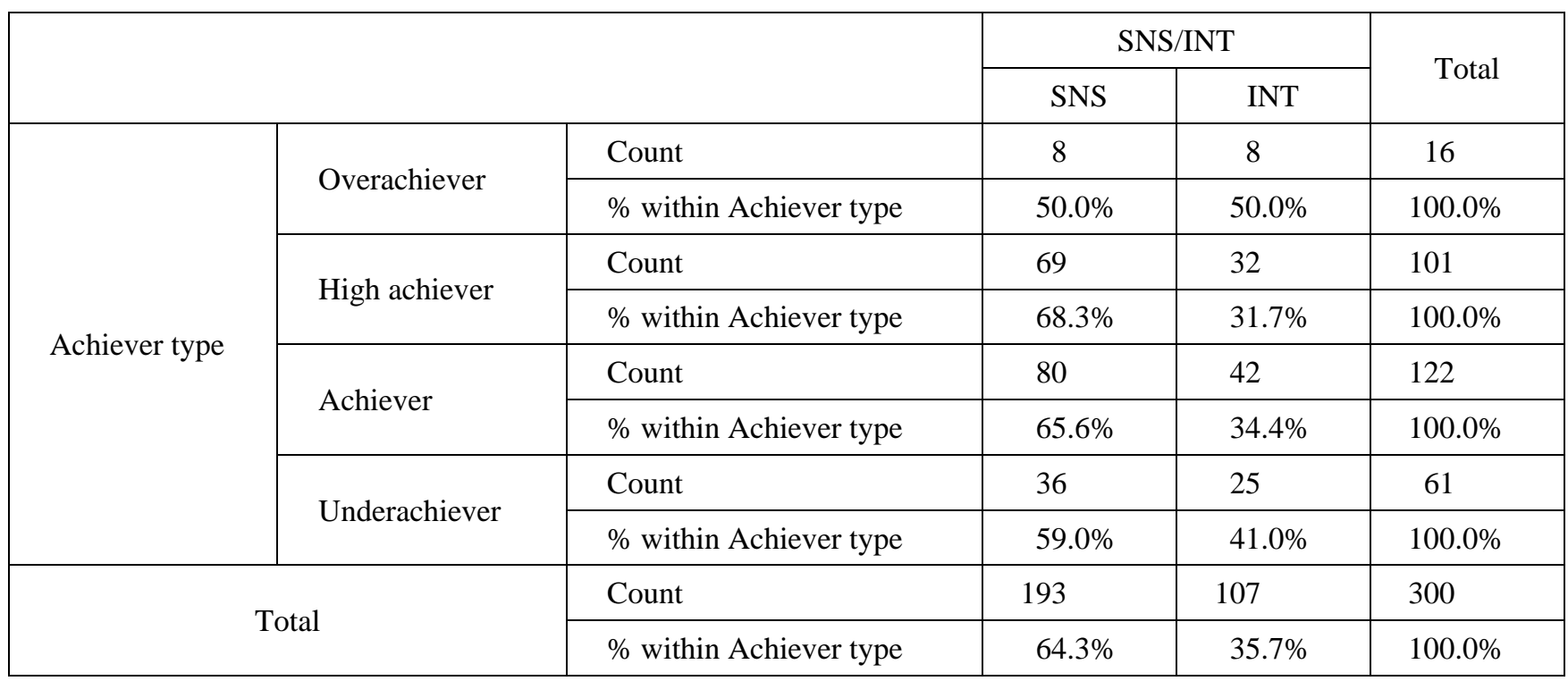

\section{Chi-Square Tests}

\begin{tabular}{|l|c|c|c|}
\hline & Value & Df & $\begin{array}{c}\text { Asymp. Sig. } \\
(2 \text {-sided) }\end{array}$ \\
\hline Pearson Chi-Square & $2.964^{\mathrm{a}}$ & 3 & .397 \\
\hline Likelihood Ratio & 2.902 & 3 & .407 \\
\hline N of Valid Cases & 300 & & \\
\hline
\end{tabular}

a. 0 cells $(.0 \%)$ have expected count less than 5. The minimum expected count is 5.71 .

The $\mathrm{X}_{\text {calc }}^{2}$ is less than 7.81 so result is statistically insignificant

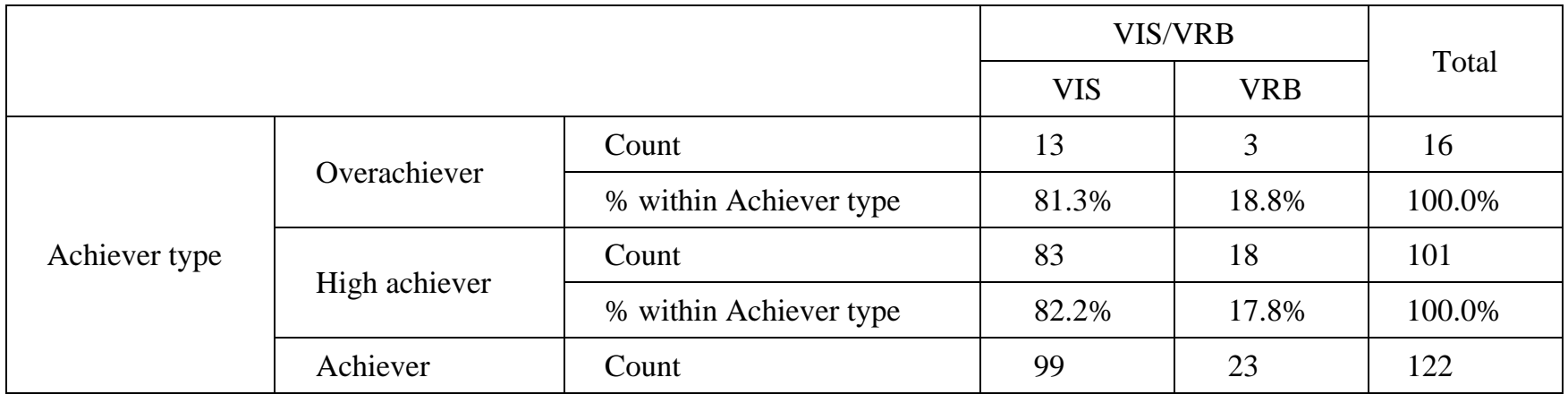




\begin{tabular}{|l|l|l|l|l|l|}
\hline & & $\%$ within Achiever type & $81.1 \%$ & $18.9 \%$ & $100.0 \%$ \\
\cline { 2 - 5 } & \multirow{3}{*}{ Underachiever } & Count & 47 & 14 & 61 \\
\cline { 2 - 5 } & $\%$ within Achiever type & $77.0 \%$ & $23.0 \%$ & $100.0 \%$ \\
\hline \multirow{2}{*}{ Total } & Count & 242 & 58 & 300 \\
\cline { 2 - 5 } & $\%$ within Achiever type & $80.7 \%$ & $19.3 \%$ & $100.0 \%$ \\
\hline
\end{tabular}

\section{Chi-Square Tests}

\begin{tabular}{|l|c|c|c|}
\hline & Value & Df & $\begin{array}{c}\text { Asymp. Sig. } \\
(2 \text {-sided })\end{array}$ \\
\hline Pearson Chi-Square & $.681^{\mathrm{a}}$ & 3 & .878 \\
\hline Likelihood Ratio & .663 & 3 & .882 \\
\hline N of Valid Cases & 300 & & \\
\hline
\end{tabular}

a. 1 cells $(12.5 \%)$ have expected count less than 5. The minimum expected count is 3.09 .

The $\mathbf{X}^{2}$ calc is less than 7.81 so result is statistically insignificant

\begin{tabular}{|c|c|c|c|c|c|}
\hline & & & \multicolumn{2}{|c|}{ SEQ/GLO } & \multirow{2}{*}{ Total } \\
\hline & & & SEQ & GLO & \\
\hline \multirow{8}{*}{ Achiever type } & \multirow{2}{*}{ Overachiever } & Count & 12 & 4 & 16 \\
\hline & & $\%$ within Achiever type & $75.0 \%$ & $25.0 \%$ & $100.0 \%$ \\
\hline & \multirow{2}{*}{ High achiever } & Count & 63 & 38 & 101 \\
\hline & & $\%$ within Achiever type & $62.4 \%$ & $37.6 \%$ & $100.0 \%$ \\
\hline & \multirow{2}{*}{ Achiever } & Count & 81 & 41 & 122 \\
\hline & & $\%$ within Achiever type & $66.4 \%$ & $33.6 \%$ & $100.0 \%$ \\
\hline & \multirow{2}{*}{ Underachiever } & Count & 35 & 26 & 61 \\
\hline & & $\%$ within Achiever type & $57.4 \%$ & $42.6 \%$ & $100.0 \%$ \\
\hline \multirow{2}{*}{\multicolumn{2}{|c|}{ Total }} & Count & 191 & 109 & 300 \\
\hline & & $\%$ within Achiever type & $63.7 \%$ & $36.3 \%$ & $100.0 \%$ \\
\hline
\end{tabular}

\section{Chi-Square Tests}

\begin{tabular}{|l|c|c|c|}
\hline & Value & Df & $\begin{array}{c}\text { Asymp. Sig. } \\
(2 \text {-sided })\end{array}$ \\
\hline Pearson Chi-Square & $2.396^{\mathrm{a}}$ & 3 & .494 \\
\hline Likelihood Ratio & 2.431 & 3 & .488 \\
\hline N of Valid Cases & 300 & & \\
\hline
\end{tabular}

a. 0 cells $(.0 \%)$ have expected count less than 5. The minimum expected count is 5.81 . 
The $\mathrm{X}^{2}$ calc is less than 7.81 so result is statistically insignificant

\begin{tabular}{|c|c|c|c|c|c|}
\hline & & & & & \\
\hline & & & ACT & REF & Trat \\
\hline & & Count & 64 & 68 & 132 \\
\hline Condor & ivrate & $\%$ within Gender & $48.5 \%$ & $51.5 \%$ & $100.0 \%$ \\
\hline 与onus & Femole & Count & 87 & 81 & 168 \\
\hline & гепाале & $\%$ within Gender & $51.8 \%$ & $48.2 \%$ & $100.0 \%$ \\
\hline & & Count & 151 & 149 & 300 \\
\hline & & $\%$ within Gender & $50.3 \%$ & $49.7 \%$ & $100.0 \%$ \\
\hline
\end{tabular}

\begin{tabular}{|c|c|c|c|c|c|}
\hline & & & & & \\
\hline & & & SNS & INT & Trat \\
\hline & S & Count & 87 & 45 & 132 \\
\hline$C$ & IVIale & $\%$ within Gender & $65.9 \%$ & $34.1 \%$ & $100.0 \%$ \\
\hline 与стис- & Боm & Count & 106 & 62 & 168 \\
\hline & รетат & $\%$ within Gender & $63.1 \%$ & $36.9 \%$ & $100.0 \%$ \\
\hline & & Count & 193 & 107 & 300 \\
\hline & & $\%$ within Gender & $64.3 \%$ & $35.7 \%$ & $100.0 \%$ \\
\hline
\end{tabular}

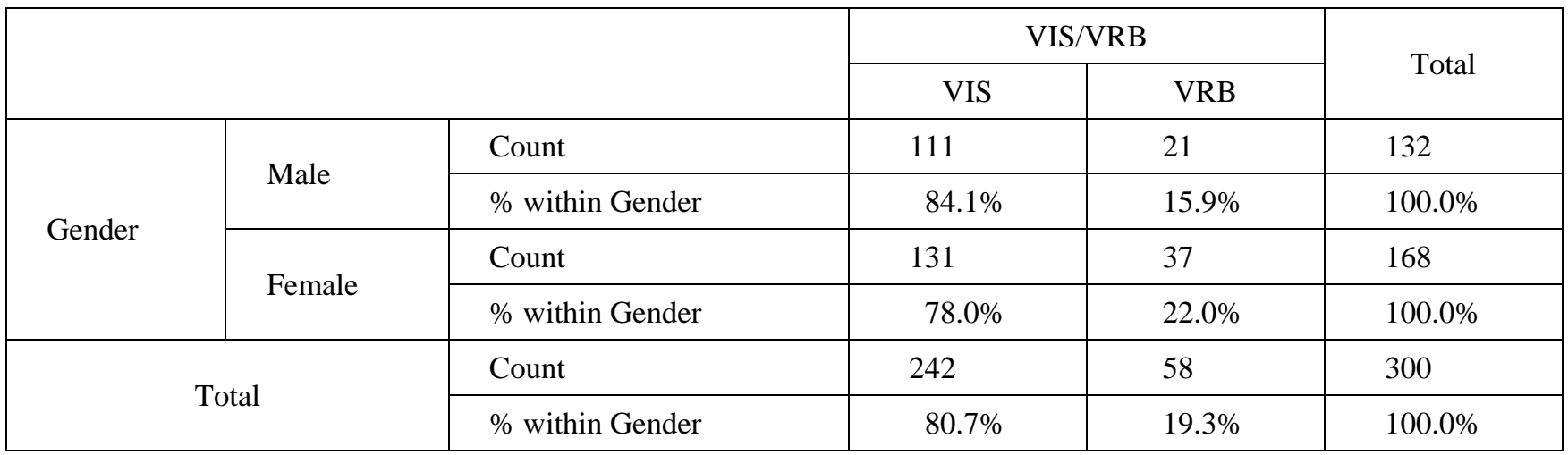

\begin{tabular}{|c|c|c|c|c|c|}
\hline & & & \multicolumn{2}{|c|}{ SEQ/GLO } & \multirow{2}{*}{ Total } \\
\hline & & & SEQ & GLO & \\
\hline \multirow{4}{*}{ Gender } & \multirow{2}{*}{ Male } & Count & 78 & 54 & 132 \\
\hline & & $\%$ within Gender & $59.1 \%$ & $40.9 \%$ & $100.0 \%$ \\
\hline & \multirow{2}{*}{ Female } & Count & 113 & 55 & 168 \\
\hline & & $\%$ within Gender & $67.3 \%$ & $32.7 \%$ & $100.0 \%$ \\
\hline \multirow{2}{*}{\multicolumn{2}{|c|}{ Total }} & Count & 191 & 109 & 300 \\
\hline & & $\%$ within Gender & $63.7 \%$ & $36.3 \%$ & $100.0 \%$ \\
\hline
\end{tabular}




\section{Association between Inter - gender Exam Performances}

Achiever type * Gender Cross - tabulation

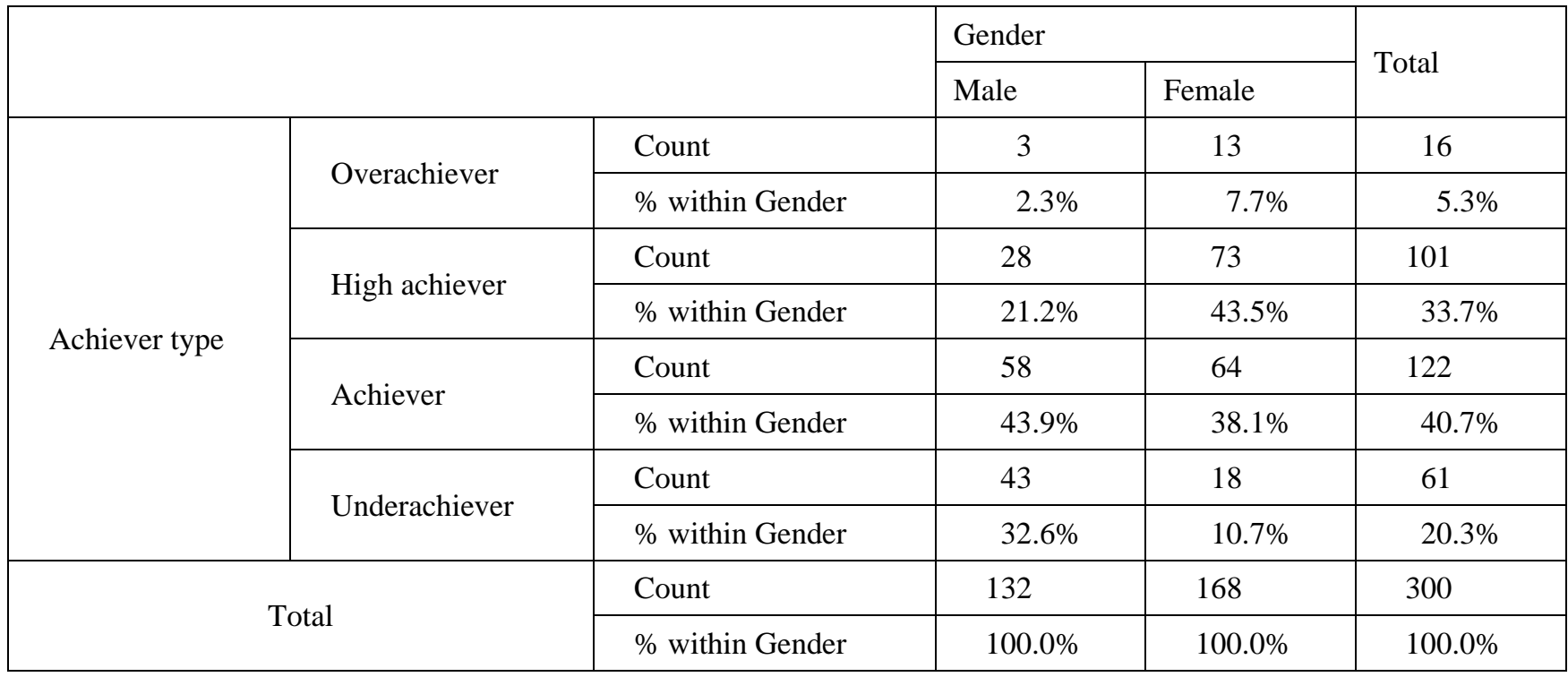

Chi-Square Tests

\begin{tabular}{|l|c|c|c|}
\hline & Value & Df & $\begin{array}{c}\text { Asymp. Sig. } \\
\text { (2-sided) }\end{array}$ \\
\hline Pearson Chi-Square & $32.996^{\mathrm{a}}$ & 3 & .000 \\
\hline Likelihood Ratio & 34.029 & 3 & .000 \\
\hline N of Valid Cases & 300 & & \\
\hline
\end{tabular}

a. 0 cells $(0.0 \%)$ have expected count less than 5. The minimum expected count is 7.04 .

The $\mathrm{X}^{2}$ calc is more than 7.81 so result is statistically significant

\section{Class Preponderance}

A greater number of $4^{\text {th }}$ year students were Reflective as opposed to relatively higher Active learning style seen in $3^{\text {rd }}$ and final year. All three years inclined more towards Sensing, Visual and Sequential learning types.

\begin{tabular}{|c|c|c|c|c|c|}
\hline & & & \multicolumn{2}{|c|}{$\mathrm{ACT} / \mathrm{REF}$} & \multirow{2}{*}{ Total } \\
\hline & & & $\mathrm{ACT}$ & REF & \\
\hline \multirow{6}{*}{ Class } & \multirow{2}{*}{ 3rd year MBBS } & Count & 51 & 49 & 100 \\
\hline & & $\%$ within Class & $51.0 \%$ & $49.0 \%$ & $100.0 \%$ \\
\hline & \multirow{2}{*}{ 4th year MBBS } & Count & 45 & 55 & 100 \\
\hline & & $\%$ within Class & $45.0 \%$ & $55.0 \%$ & $100.0 \%$ \\
\hline & \multirow{2}{*}{ 5th year MBBS } & Count & 55 & 45 & 100 \\
\hline & & $\%$ within Class & $55.0 \%$ & $45.0 \%$ & $100.0 \%$ \\
\hline & \multirow{2}{*}{ Total } & Count & 151 & 149 & 300 \\
\hline & & $\%$ within Class & $50.3 \%$ & $49.7 \%$ & $100.0 \%$ \\
\hline
\end{tabular}




\begin{tabular}{|c|c|c|c|c|c|}
\hline & & & \multicolumn{2}{|c|}{ VIS/VRB } & \multirow{2}{*}{ Total } \\
\hline & & & VIS & VRB & \\
\hline \multirow{6}{*}{ Class } & \multirow{2}{*}{ 3rd year MBBS } & Count & 82 & 18 & 100 \\
\hline & & $\%$ within Class & $82.0 \%$ & $18.0 \%$ & $100.0 \%$ \\
\hline & \multirow{2}{*}{ 4th year MBBS } & Count & 87 & 13 & 100 \\
\hline & & $\%$ within Class & $87.0 \%$ & $13.0 \%$ & $100.0 \%$ \\
\hline & \multirow{2}{*}{ 5th year MBBS } & Count & 73 & 27 & 100 \\
\hline & & $\%$ within Class & $73.0 \%$ & $27.0 \%$ & $100.0 \%$ \\
\hline & \multirow{2}{*}{ Total } & Count & 242 & 58 & 300 \\
\hline & & $\%$ within Class & $80.7 \%$ & $19.3 \%$ & $100.0 \%$ \\
\hline
\end{tabular}

\begin{tabular}{|c|c|c|c|c|c|}
\hline & & & \multicolumn{2}{|c|}{ SNS/INT } & \multirow{2}{*}{ Total } \\
\hline & & & SNS & INT & \\
\hline \multirow{6}{*}{ Class } & \multirow{2}{*}{ 3rd year MBBS } & Count & 69 & 31 & 100 \\
\hline & & $\%$ within Class & $69.0 \%$ & $31.0 \%$ & $100.0 \%$ \\
\hline & \multirow{2}{*}{ 4th year MBBS } & Count & 61 & 39 & 100 \\
\hline & & $\%$ within Class & $61.0 \%$ & $39.0 \%$ & $100.0 \%$ \\
\hline & \multirow{2}{*}{ 5th year MBBS } & Count & 63 & 37 & 100 \\
\hline & & $\%$ within Class & $63.0 \%$ & $37.0 \%$ & $100.0 \%$ \\
\hline & \multirow{2}{*}{ Total } & Count & 193 & 107 & 300 \\
\hline & & $\%$ within Class & $64.3 \%$ & $35.7 \%$ & $100.0 \%$ \\
\hline
\end{tabular}

\begin{tabular}{|c|c|c|c|c|c|}
\hline & & & \multicolumn{2}{|c|}{ SEQ/GLO } & \multirow{2}{*}{ Total } \\
\hline & & & SEQ & GLO & \\
\hline \multirow{6}{*}{ Class } & \multirow{2}{*}{ 3rd year MBBS } & Count & 66 & 34 & 100 \\
\hline & & $\%$ within Class & $66.0 \%$ & $34.0 \%$ & $100.0 \%$ \\
\hline & \multirow{2}{*}{ 4th year MBBS } & Count & 66 & 34 & 100 \\
\hline & & $\%$ within Class & $66.0 \%$ & $34.0 \%$ & $100.0 \%$ \\
\hline & \multirow{2}{*}{ 5th year MBBS } & Count & 59 & 41 & 100 \\
\hline & & $\%$ within Class & $59.0 \%$ & $41.0 \%$ & $100.0 \%$ \\
\hline & \multirow{2}{*}{ Total } & Count & 191 & 109 & 300 \\
\hline & & $\%$ within Class & $63.7 \%$ & $36.3 \%$ & $100.0 \%$ \\
\hline
\end{tabular}

\section{Discussion}

Preponderance of learning strategies among Engineering majors and Medical students was found in the past but no such study of this type has ever been conducted in the context of Pakistan. Our study goes a step further in order to find the association between exam per- 
formance and academic learning strategies. However, no statistical significance was found between exam performance and academic learning strategies. Exam performance is multifactorial. Probable reasons explaining the results of our study coming out negative could be difference in intellectual capacity of students, different approach towards examination, poor grading system, difference in dedication towards studies, peer pressure, diversity in strength of foundation in basic sciences and varying attitude of students towards teachers. Certain policy measures to boost academic performance would involve uniform exam writing, replacement of pass/fail criteria by Grade Point Average (GPA) and opting channels for proper counseling of students. However, some factors were not accounted for - IQ being one of them. Further limitations include our study being conducted only on students from King Edward Medical University. Moreover, only Index Learning of Styles (ILS) was used to determine learning strategies. Other models were not followed. For further studies, other models can also be followed to evaluate learning strategies. Medical students hailing from different institutions can be researched upon. Factors like IQ should be controlled in other studies. An ILS model for teaching strategies should be conducted among teachers and compatibility established with predominant learning strategies shown by our research.

\section{Conclusion}

The Null Hypothesis that states that there is no association between academic learning strategies and annual examination results among medical students of King Edward Medical University cannot be rejected. Our research establishes that females outperform their male counterparts in academic performance.

\section{References}

1. Nuzhat A, Salem RO, Qadri M, Al-Hamdan N. Learning style preferences of medical students: a single institute experience from Saudi Arabia. Int J Med Educ [online] 2011 Aug 01 [cited 2013 Mar 03]; 2:70-73. Available from:

http://www.ijme.net/archive/2/students-learning-stylepreferences.pdf

2. Su AY. Relationships between the Learning-style Preferences and the Characteristics and Academic Performance of Taiwanese College Hospitality Students. J Hum Res Adu Learn [online] 2012 Dec [cited 2013 Mar 03]; 8 (2): 158-166. Available from: http://hraljournal.com/Page/19\%20Yen\%20Lun\%20Su2.pdf

3. Holley JH, Jenkins EK. The relationship between student learning style and performance on various test question formats. J Educ Bus [online] 1993 May [cited 2013 Mar 03]; 68 (5): 301-308. Available from: http://eric.ed.gov/?id=EJ463540

4. Fleming N, Baume D. Learning Styles Again: VAR King up the right tree! Educ Develop. [online] 2006 Nov [cited 2013 Mar 03]; 7(4):4-7. Available from: http://www.johnsilverio.com/EDUI6702/Fleming_VAR K_learningstyles.pdf

5. Felder RM, Silverman LK. Learning and teaching styles in engineering education. Engr education [online] 1988 [cited 2013 Mar 03]; 78 (7): 674-681. Available from: http://www4.ncsu.edu/unity/lockers/users/f/felder /public/Papers/LS-1988.pdf

6. Viola SR, Graf S, Leo T. Investigating relationships within the Index of Learning Styles: a data driven approach. Inter Tech Smart Educ [online] 2007 Feb [cited 2013 Mar 03]; 4 (1): 7-18. Available from: http://wit.tuwien.ac.at/people/graf/publications/viola_gr af_kinshuk_leo_ITSE4-1-2.pdf

7. Vermunt JD. The regulation of constructive learning processes. Brit J Educpsyc [online] 1998 [cited 2015 Mar 03]; 68 (2): 149-171. Available from: http://onlinelibrary.wiley.com/doi/10.1111/j.20448279.1998.tb01281.x/abstract

8. Kolmos, A. and Holgaard, J. E. Learning Styles of Science and Engineering Students in Problem and Project Based Education. In Book of Abstracts. Sense Publishers, 2008.

9. Lujan HL, DiCarlo SE. First - year medical students pre-fer multiple learning styles. Adv Phys Educ [online] 2006 Mar 1 [cited 2015 Mar 03]; 30 (1): 13-16. Available from:

http://advan.physiology.org/content/30/1/13.fulltext.pdf + html 